\section{RSP}

http://www.rsp.fsp.usp.br/
Revista de Saúde Pública

\title{
Por que a assistência em saúde mental não acompanha a estruturação da atenção primária?
}

\author{
Antonio Moacir de Jesus Lima' (iD, Eli lola Gurgel Andrade" (iD, Antonio Thomaz Gonzaga da \\ Matta Machado"II ID, Alainer de Fátima dos Santos" (iD \\ I Universidade Federal dos Vales do Jequitinhonha e Mucuri. Faculdade de Ciências Biológicas e da Saúde. \\ Departamento de Enfermagem. Diamantina, MG, Brasil \\ " Universidade Federal de Minas Gerais. Faculdade de Medicina. Programa de Pós-Graduação em Saúde \\ Pública. Belo Horizonte, MG, Brasil \\ III Universidade Federal de Minas Gerais. Faculdade de Medicina. Departamento de Medicina Preventiva e \\ Social. Belo Horizonte, MG, Brasil
}

\author{
Correspondência: \\ Antonio Moacir de Jesus Lima \\ Rua João Evangelista da Rocha, 606 - \\ Vila Operária \\ 39100-000 Diamantina, MG, Brasi \\ E-mail: antonio.moacir@ufvjm.edu.br
}

Recebido: 24 out 2020

Aprovado: 12 mar 2021

Como citar: Lima AMJ, Andrade EIG, Mata-Machado AT, Santos AF. Por que a assistência em saúde mental não acompanha a estruturação da atenção primária? Rev Saude Publica. 2021;55:99. https://doi.org/10.11606/ s1518-8787.2021055003344

Copyright: Este é um artigo de acesso aberto distribuído sob os termos da Licença de Atribuição Creative Commons, que permite uso irrestrito, distribuição e reprodução em qualquer meio desde que o autor e a fonte originais sejam creditados.

\section{RESUMO}

OBJETIVO: Verificar se equipes de atenção básica que possuem atributos mais bem estruturados da atenção primária à saúde (APS) conseguem oferecer melhor assistência em saúde mental (SM).

MÉTODOS: Estudo transversal realizado a partir dos dados da avaliação externa do segundo ciclo do Programa de Melhoria do Acesso e da Qualidade da Atenção Básica (PMAQ-AB), envolvendo 31.587 equipes de atenção básica, entre 2013 e 2014. Foram construídas duas tipologias: qualidade da assistência em saúde mental (variável dependente) e estruturação da APS segundo atributos essenciais (variável independente). Para a construção da tipologia de saúde mental, foram identificados conteúdos sobre o tema no módulo II do PMAQ e realizados somatórios das perguntas para a categorização dos índices. Para a estruturação da APS segundo atributos, utilizou-se técnica Delphi para consensualidade em quatro rodadas referendadas por especialistas. Com análises de regressão logística multinomial, verificou-se associação entre as tipologias e identificou-se qual atributo mais contribuía para qualidade da atenção em saúde mental.

RESULTADOS: Verificou-se que $29,2 \%$ das equipes encontram-se em um nível baixo de qualidade em assistência à SM, enquanto 7,5\% das equipes apresentam um nível baixo de estruturação da APS segundo atributos essenciais. Diferenças regionais são mantidas, tanto para estruturação da APS quanto para a qualidade da assistência à saúde mental. Evidenciou-se uma chance maior de realizar uma assistência em SM com melhor qualidade quando a APS está mais bem estruturada em nível alto $(\mathrm{OR}=14,74)$ e em nível médio $(\mathrm{OR}=2,193)$. Alto nível de Integralidade está associado a alto nível de Qualidade da Assistência em SM (OR = 3,21).

CONCLUSÕES: Os resultados indicam que há predomínio de baixos níveis de qualidade da assistência à saúde mental, em descompasso com o processo de estruturação da APS, considerando seus atributos essenciais.

DESCRITORES: Assistência à Saúde Mental. Atenção Primária à Saúde. Qualidade da Assistência à Saúde. Administração de Serviços de Saúde. 


\section{INTRODUÇÃO}

Em todo o mundo, os transtornos mentais representam uma parcela elevada dos registros de doenças, só nas Américas, um estudo ${ }^{1}$ revelou prevalência de $17 \%$, sendo $22,5 \%$ das doenças na América do Norte e 14,8\% em toda América Latina. No Brasil, os transtornos mentais também apresentam taxa considerável 29,5\%, tendo a ansiedade, 19,9\%, e os transtornos de humor, $11 \%$, como os mais prevalentes, seguidos pelos transtornos de controle de impulso, $4,2 \%$, e outros, decorrentes do uso de substâncias psicoativas, $3,6 \%$, números que fizeram o país pular da sexta para a terceira posição ${ }^{2}$ no estudo de Carga Global de Doença (Global Burden of Disease - GBD), entre 1990 e 2015.

A triagem para detecção e tratamento precoce realizada na atenção primária em saúde (APS) pode melhorar a qualidade de vida dos pacientes, ajudar no melhor uso dos investimentos em assistência à saúde e reduzir significativamente as complicações e comorbidades médicas ${ }^{3}$. Assim, a saúde mental (SM) deve ser entendida como um campo de intervenção prioritário para as equipes da Estratégia de Saúde da Família (ESF), devido a sua atuação próxima à comunidade, não apenas nas unidades de saúde, como também no espaço social em que vivem e circulam os usuários.

Contudo, a inserção da saúde mental na APS encontra dificuldades em alguns aspectos, como demonstram diversos estudos. Um desses entraves está na formação profissional, pois os trabalhadores desenvolviam suas ações pautados no modelo biológico, com intervenções centradas na consulta clínica, na medicalização e no diagnóstico, visando apenas à remissão dos sintomas ${ }^{4}$. Outros estudos reforçam essa percepção e apontam para a ausência de corresponsabilização em encaminhamentos desnecessários e uma importante fragilidade na capacidade de identificação de casos por parte dos profissionais da $\mathrm{ESF}^{5,6}$. Mais recentemente, outro estudo apontou que mais de $60 \%$ dos profissionais da APS afirmaram sentir-se despreparados para acolherem as demandas de saúde mental?

Outro empecilho para essa inserção vincula-se ao processo de trabalho das equipes, pois, ainda que algumas práticas sejam inovadoras, ainda não se identificam as mudanças necessárias para humanização do cuidado às pessoas com sofrimento psíquico ${ }^{8}$. Contudo, esse processo ainda está em fase de implementação e, portanto, necessita de ampliação do acesso, por meio da melhoria da assistência ${ }^{5}$.

Essa ampliação do acesso passa também por questões de infraestrutura, tanto das unidades básicas e quanto de recursos humanos, e de desestruturação da rede de atenção, que ainda mantém uma organização hierárquica e uma dinâmica comunicacional pautada em protocolos técnicos de saúde . Em Salvador, Bahia, por exemplo, um estudo apontou uma dependência da Rede de Atenção Psicossocial (RAPS) do hospital psiquiátrico, apontando para a necessidade de ações que promovam o fortalecimento da rede, sua articulação e desinstitucionalização do Centro de Atenção Psicossocial (CAPS) ${ }^{10}$. Em Fortaleza, Ceará, evidenciou-se a prática de medicalização aos transtornos mentais como forma de mitigar as dificuldades de acesso ao CAPS enfrentadas pelos pacientes ${ }^{11}$.

No entanto o engajamento dos trabalhadores tem resultado em experiências bem-sucedidas em diversas partes do Brasil, contatando-se o avanço na articulação entre a saúde mental e a atenção primária, com mais de $56 \%$ das equipes realizando ações nessa área ${ }^{12}$, como de promoção de ações de SM relacionadas ao apoio matricial (AM), ao trabalho em rede, diversidade de práticas e participação social. O matriciamento poderia aumentar a resolutividade e a efetividade das ações da ESF, com a atuação conjunta entre especialistas e profissionais das equipes no território. As experiências conhecidas ${ }^{13,14}$ potencializaram a construção coletiva de saberes e melhoria na comunicação entre os trabalhadores, os usuários e os gestores, além de ampliar os conhecimentos sobre saúde mental e maior corresponsabilização, o que contribui para a identificação e o acolhimento dos casos e construção de projetos terapêuticos singulares. 
Outras formas de enfrentamento também estão em curso $^{9,12}$ e mostrando resultados, por exemplo, em Salvador, onde tem sido praticada a Internação Domiciliar, realizada pelas equipes da ESF para integrar as ações de SM na APS, e em Ribeirão Preto, onde têm-se optado pelas Visitas Domiciliares, que contribuem para postergar a internação psiquiátrica por meio do acompanhamento dos egressos, orientações aos familiares e demais esforços para evitar a medicalização do sofrimento psíquico.

As ações de SM desenvolvidas na APS contribuem para as transformações no paradigma da assistência psiquiátrica, determinando a desconstrução do histórico distanciamento entre as práticas psiquiátricas excludentes e a atenção primária. Assim, reorientam o modelo da assistência prestada aos portadores de sofrimento mental com dispositivos comunitários e se configuram como uma realidade para implementação da Política Nacional de Saúde Mental ${ }^{12}$.

Esse conjunto de desafios e experiências significativas indica que a inserção de ações de SM na APS constitui um amplo campo de possibilidades e de complexas questões. Este estudo busca contribuir para avaliar essa inserção e objetivou analisar se as equipes de atenção básica que apresentam atributos da APS mais bem estruturados conseguem oferecer melhor assistência na área da Saúde Mental.

\section{MÉTODOS}

Estudo transversal realizado a partir dos dados do segundo ciclo do PMAQ-AB, ocorrido nos anos de 2013 e 2014, envolvendo 31.587 Equipes de Atenção Básica (EqAB), de todas as regiões do país. Para esse estudo, foram utilizados os dados contidos no módulo II, referente à avaliação externa e relacionado ao processo de trabalho das equipes, em que as perguntas são respondidas pelo coordenador e contém 750 perguntas. A coleta de dados do PMAQ-AB foi coordenada por um grupo de pesquisadores de universidades e instituições de pesquisa responsáveis pela avaliação externa, que treinou e acompanhou os entrevistadores de campo e supervisores da coleta de dados.

Fizeram parte do estudo $31.587 \mathrm{EqAB}$, no entanto, foram excluídas 1.809 equipes por motivos diversos: 713 não participaram de todo o ciclo de avaliação; 353 não cumpriram com os compromissos assumidos na contratualização e 743 obtiveram nota zero. O universo final analisado contemplou 29.778 equipes.

A partir desses dados, foram elaboradas duas tipologias: Estruturação da APS, a partir dos seus atributos essenciais (variável independente); e Qualidade da Assistência prestada na área da Saúde Mental (variável dependente). Na primeira tipologia utilizou-se a técnica Delphi para a consensualização das perguntas que poderiam compor cada atributo. Todos os itens do questionário foram condensados em duzentas e cinco (205) perguntas e, assim, foram enviadas para cinco pesquisadores com titulação de doutorado em Saúde Coletiva, autores de publicações em periódicos de referência nacional para o tema e vinculados a instituições de ensino superior em diferentes estados. $\mathrm{O}$ objetivo era que esses cientistas indicassem a qual atributo cada pergunta se referia. Esse processo excluiu 70 perguntas: 35 delas, os juízes definiram como "não se aplica" e as outras 35 faziam referência à SM e foram eliminadas para evitar colinearidade. Assim, foi possível construir um consenso, após quatro rodadas, permitindo isoladamente a avaliação de cada um dos quatro atributos essenciais da APS.

O Quadro expressa de forma sintética a relação entre cada Atributo Essencial da APS e os conteúdos das questões utilizadas no PMAQ-AB, de acordo com o consenso definido pelos especialistas.

Para atipologia da Qualidade da atenção prestada em saúde mental, foramidentificadas 35 perguntas no formulário II PMAQ-AB, quecontemplam o acolhimento, a consulta, o acompanhamento, a solicitação de exames, a prescrição de psicotrópicos, o encaminhamento para os serviços especializados e a abordagem multiprofissional dos trabalhadores do NASF com as equipes. Também foram realizadas perguntas mais detalhadas sobre o processo de trabalho dos profissionais,, envolvendo tratamento, promoção, prevenção, redução de danos e a reabilitação da saúde aos 
Quadro. Lista de conteúdos do PMAQ-AB relacionados a cada atributo essencial da APS - consenso dos especialistas.

\begin{tabular}{|c|c|}
\hline Atributo essencial & Conteúdo condensado das questões utilizadas no PMAQ \\
\hline Primeiro contato & $\begin{array}{l}\text { - Estruturação da área de abrangência da equipe; } \\
\text { - Atendimento da demanda espontânea; } \\
\text { - Visitas domiciliares; } \\
\text { - Atividades relativas ao agendamento de consultas; } \\
\text { - Acolhimento e tempo de espera na unidade; } \\
\text { - Organização da agenda; } \\
\text { - Atenção às comunidades tradicionais; assentados; população rural; } \\
\text { - Existência de transporte. }\end{array}$ \\
\hline Longitudinalidade & $\begin{array}{l}\text { - Manejo do telessaúde; } \\
\text { - Prontuários; } \\
\text { - Formulários e registro das ações realizadas; } \\
\text { - Satisfação do usuário; } \\
\text { - Canais de comunicação com a população; } \\
\text { - Controle social. }\end{array}$ \\
\hline Integralidade & $\begin{array}{l}\text { - Educação permanente e capacitações; } \\
\text { - Atividades de planejamento; } \\
\text { - Disponibilidade de informações; } \\
\text { - Apoio institucional; } \\
\text { - Execução das atividades planejadas; } \\
\text { - Articulação do trabalho com as possibilidades locais; } \\
\text { - Estrutura, insumos e instrumentalização das ações; } \\
\text { - Encaminhamento de demandas; } \\
\text { - Processo de trabalho da equipe; } \\
\text { - Atenção ao tabagismo, tuberculose e hanseníase; } \\
\text { - PICS e práticas corporais; } \\
\text { - Promoção e educação em saúde; } \\
\text { - Programas Bolsa Família e Saúde na Escola; } \\
\text { - Cuidado domiciliar; } \\
\text { - Estrutura do NASF. }\end{array}$ \\
\hline Coordenação do cuidado & $\begin{array}{l}\text { - Articulação com IES; } \\
\text { - Monitoramento e Controle das ações desenvolvidas; } \\
\text { - Avaliação de resultados; } \\
\text { - Suporte para complexidades; } \\
\text { - Apoio matricial; } \\
\text { - Contrarreferência; } \\
\text { - Funcionamento do NASF; } \\
\text { - Instrumentos de avaliação; } \\
\text { - Gestão dos casos graves; } \\
\text { - Controle e avaliação dos programas desenvolvidos. }\end{array}$ \\
\hline
\end{tabular}

PMAQ: Programa de Melhoria do Acesso e da Qualidade. PICS: Práticas Integrativas Complementares em Saúde. NASF: Núcleo Ampliado de Saúde da Família. IES: instituição de ensino superior.

portadores de sofrimento psíquico e aos usuários de álcool e outras drogas. Construídas as duas tipologias, as questões foram somadas e os índices, categorizados (Quadro).

As duas tipologias (atributos e saúde mental) tiveram seus índices categorizados em três cenários, para expressar os níveis baixo (0-32,99\%), médio (33-65,99\%) e alto (66-100\%). Os atributos essenciais expressos em sua categoria são Integralidade, Coordenalção, Longitudinalidade e Primeiro Contato, além ainda de uma variável geral, a APS, resultante da soma das outra quatro que compõem essa tipologia. Para efeito de pontuação, as 18 variáveis politômicas da segunda tipologia foram recodificadas como dicotômicas em suas respostas ( 1 = sim e 0 = não/não sabe/não se aplica/não respondeu).

$\mathrm{Na}$ análise descritiva, as EqAB foram distribuídas conforme a estruturação da assistência em saúde mental, os atributos essenciais e geral da APS, além da classificação por regiões. Para investigar as associações, foram realizadas análises de regressão logística multinomial, tendo como variável dependente a qualidade da assistência prestada em SM e, como variável independente, os atributos da APS. O cenário de Baixa Estruturação de SM foi assumido como categoria de referência e, portanto, as categorias das tipologias foram dicotomizadas em baixo e médio/alto.

A magnitude das associações foi representada pela razão de chances odds ratio (OR), com respectivo Intervalo de Confiança de 95\% (IC95\%) e nível de significância de 5\% ( $\mathrm{p} \leq 0,05$ ). Utilizou-se o programa SPSS Statistics 20 para realizar todas as análises estatísticas. 
O estudo cumpre as normas e diretrizes regulamentadoras de pesquisa envolvendo seres humanos da Resolução 466/2012, com aprovação do Comitê de Ética em Pesquisa da Universidade Federal de Minas Gerais, com o Registro 28.804, em 30/5/2012.

\section{RESULTADOS}

Na distribuição da qualidade dos cuidados em Saúde Mental (Tabela 1), por níveis e regiões, observa-se que 52,1\% das equipes se encontram no nível médio, 29,2\% no nível baixo e uma pequena parcela no nível alto $18,7 \%$.

A região Sudeste apresentou maior número de equipes de nível alto (23,7\%) e o menor número de nível baixo (23,7\%), enquanto a região Norte tem os índices inversos: 52,9\% das equipes de nível baixo e 7\% de nível alto.

Quando a distribuição é feita por estruturação da APS, níveis e regiões (Tabela 2), a maioria das equipes situa-se no nível médio de estruturação da APS (86,52\%), 7,5\% estão no nível baixo e apenas 5,93\% se encontram no nível alto de estruturação.

Quanto à distribuição regional, a região Sudeste mais uma vez apresentou o maior número de equipes de alto nível (7,5\%), enquanto a região Nordeste apresenta o menor índice de EqAB no nível baixo (5,9\%). A Região Norte destaca-se negativamente por apresentar os piores índices em todos os níveis, com o maior número de equipes de nível baixo (16,9\%) e menor quantidade de esquipes de nível alto $(1,5 \%)$.

Na distribuição da estruturação da APS segundo os atributos essenciais, por níveis (Tabela 3), destaca-se o atributo Longitudinalidade destaca-se que apresenta 36\% das EqAB de nível alto. Em contrapartida, o atributo Coordenação possui os piores índices (28\% de nível baixo e $0,7 \%$ de nível alto);

Tabela 1. Distribuição da qualidade de atenção em saúde mental, por níveis e regiões, Brasil PMAQ-AB 2014.

\begin{tabular}{|c|c|c|c|c|c|c|}
\hline \multirow{3}{*}{ Regiões } & \multicolumn{6}{|c|}{ Níveis } \\
\hline & \multicolumn{2}{|c|}{ Baixo } & \multicolumn{2}{|c|}{ Médio } & \multicolumn{2}{|c|}{ Alto } \\
\hline & $\mathbf{n}$ & $\%$ & $\mathbf{n}$ & $\%$ & $\mathbf{n}$ & $\%$ \\
\hline Sul & 1.118 & 24,8 & 2.370 & 52,6 & 1.021 & 22,6 \\
\hline Sudeste & 2.288 & 22,7 & 5.416 & 53,6 & 2.396 & 23,7 \\
\hline Centro-Oeste & 933 & 41,6 & 1.082 & 48,3 & 226 & 10,1 \\
\hline Norte & 1.142 & 52,9 & 867 & 40,1 & 151 & 7 \\
\hline Nordeste & 3.210 & 29,8 & 5.774 & 53,5 & 1.784 & 16,6 \\
\hline Total & 8.691 & 29,2 & 15.509 & 52,1 & 5.578 & 18,7 \\
\hline
\end{tabular}

PMAQ-AB: Programa de Melhoria do Acesso e da Qualidade da Atenção Básica.

Tabela 2. Distribuição da estruturação da atenção primária à saúde, por regiões e níveis, Brasil PMAQ-AB 2014.

\begin{tabular}{|c|c|c|c|c|c|c|}
\hline \multirow{3}{*}{ Regiões } & \multicolumn{6}{|c|}{ Níveis } \\
\hline & \multicolumn{2}{|c|}{ Baixo } & \multicolumn{2}{|c|}{ Médio } & \multicolumn{2}{|c|}{ Alto } \\
\hline & \multicolumn{2}{|c|}{ n \% } & \multicolumn{2}{|c|}{ n \% } & \multicolumn{2}{|c|}{ n \% } \\
\hline Sul & 364 & 8,1 & 3.860 & 85,6 & 285 & 6,3 \\
\hline Sudeste & 670 & 6,6 & 8.668 & 85,8 & 762 & 7,5 \\
\hline Centro-Oeste & 209 & 9,3 & 1.961 & 87,5 & 71 & 3,2 \\
\hline Norte & 365 & 16,9 & 1.762 & 81,6 & 33 & 1,5 \\
\hline Nordeste & 638 & 5,9 & 9.514 & 88,4 & 616 & 5,7 \\
\hline Total & 2.246 & 7,55 & 25.765 & 86,52 & 1.767 & 5,93 \\
\hline
\end{tabular}

PMAQ-AB: Programa de Melhoria do Acesso e da Qualidade da Atenção Básica. 
Tabela 3. Distribuição da estruturação da atenção primária à saúde, segundo os atributos essenciais, por níveis, Brasil - PMAQ-AB 2014.

\begin{tabular}{|c|c|c|c|c|c|c|}
\hline \multirow{3}{*}{ Atributos essenciais da APS } & \multicolumn{6}{|c|}{ Níveis } \\
\hline & \multicolumn{2}{|c|}{ Baixo } & \multicolumn{2}{|c|}{ Médio } & \multicolumn{2}{|c|}{ Alto } \\
\hline & \multicolumn{2}{|c|}{ n \% } & \multicolumn{2}{|c|}{ n \% } & \multicolumn{2}{|c|}{$\mathrm{n} \%$} \\
\hline Integralidade & 2.392 & 8,0 & 24.130 & 81,0 & 3.256 & 10,9 \\
\hline Coordenação & 8.344 & 28,0 & 21.230 & 71,3 & 204 & 0,7 \\
\hline Longitudinalidade & 1.253 & 4,2 & 17.814 & 59,8 & 10.711 & 36,0 \\
\hline Primeiro contato & 4.008 & 13,5 & 25.069 & 84,2 & 701 & 2,4 \\
\hline
\end{tabular}

PMAQ-AB: Programa de Melhoria do Acesso e da Qualidade da Atenção Básica.

Tabela 4. Qualidade da assistência em SM e estruturação da APS, por atributos essenciais e indicador geral, Brasil - PMAQ-AB 2014.

\begin{tabular}{|c|c|c|c|c|c|}
\hline \multirow{3}{*}{ ESTRUTURAÇÃO da APS } & \multicolumn{5}{|c|}{ QUALIDADE DA ASSISTÊNCIA SM } \\
\hline & \multicolumn{2}{|c|}{ Baixo } & \multirow{2}{*}{$\begin{array}{c}\text { Médio } \\
\text { OR (IC95\%) }\end{array}$} & \multicolumn{2}{|r|}{ Alto } \\
\hline & OR (IC95\%) & $\mathbf{p}$ & & $\mathbf{p}$ & OR (IC95\%) \\
\hline Integralidade & 1 & 0,32 & $0,91(0,75-1,10)$ & 0,00 & $3,21(2,60-3,96)$ \\
\hline Coordenação & 1 & 0,00 & $1,42(1,27-1,59)$ & 0,00 & $2,31(1,69-3,16)$ \\
\hline Longitudinalidade & 1 & 0,87 & $0,98(0,77-1,25)$ & 0,00 & $2,25(1,74-2,90)$ \\
\hline Primeiro contato & 1 & 0,00 & $1,28(1,11-1,49)$ & 0,00 & $1,55(1,23-1,95)$ \\
\hline Indicador geral & 1 & 0,00 & $2,19(1,85-2,59)$ & 0,00 & $14,74(12,18-7,83)$ \\
\hline
\end{tabular}

PMAQ-AB: Programa de Melhoria do Acesso e da Qualidade da Atenção Básica; APS: Atenção Primária à Saúde; SM: Saúde Mental; OR: odds ratio; IC95\%: intervalo de confiança.

Na análise de associação (Tabela 4) entre a qualidade da assistência prestada em saúde mental e a estruturação da atenção primária, a partir dos atributos essenciais, por níveis, observa-se uma associação positiva em cenários de estruturação da APS de médio e alto nível. No cenário de média estruturação aumenta a chance de alcançar maior qualidade da assistência para a área de saúde mental $(\mathrm{OR}=2,193)$. Esse resultado torna-se expressivamente maior ( $\mathrm{OR}=14,742)$ quando o cenário é de alta estruturação, ou seja, quando a APS é melhor estruturada, a chance de melhores níveis de assitência chega a ser quatorze vezes maior. Associações positivas no cenário de alta estruturação da APS foram encontradas em todos os atributos (integralidade $\mathrm{OR}=3,21$; coordenação $\mathrm{OR}=2,31$; longitudinalidade $\mathrm{OR}=2,25$; primeiro contato $\mathrm{OR}=1,55)$. Por fim, para o cenário de média estruturação, apenas os atributos coordenação e primeiro contato apresentaram uma associação positiva.

\section{DISCUSSÃO}

Este estudo demonstra que há ainda um longo caminho a ser percorrido para se atingir um alto nível de qualidade do cuidado em saúde mental na APS brasileira. A maior parte das equipes encontra-se em nível médio de qualidade do cuidado ofertado e quase um terço delas apresenta nível de qualidade ruim, diferindo bastante do quadro geral de estruturação da APS no Brasil.

O número de equipes que se encontram no pior cenário para a qualidade de assistência à SM (29,2\%) é o triplo daquele encontrado para estruturação da APS. Esses resultados constatam problemas evidenciados por outros estudos que apontam o processo em curso de estruturação da APS, faltando ainda enfrentar o desafio da incorporação das práticas de saúde mental ${ }^{7,9}$.

Com efeito, quando há alta estruturação da APS também as ações em saúde mental estão bem estruturadas, realidade que alguns estudos focando centros urbanos também constataram em situações nas quais os serviços de SM já se encontram estruturados em forma de rede $\mathrm{e}^{4,10,15}$. 
Os dados evidenciaram que as regiões Sul e Sudeste possuem resultados melhores em assistência à saúde mental, enquanto as regiões Norte e Centro-Oeste apresentam os piores resultados, o que sugere que as regiões estruturam-se de diferentes formas para inserirem o cuidado de SM na APS ${ }^{7,16}$.

A análise sobre o grau de estruturação da APS constatou que poucas equipes possuem um alto grau de estruturação e uma maioria significativa encontra-se no nível médio, corroborando com os resultados de outros estudos, com diferenças percentuais ${ }^{15,17}$.

Quando se faz análise por regiões, observa-se que se mantém a relação das diferenças regionais tanto para qualidade da assistência em SM quanto para estruturação da APS, indicando que a região norte obteve os piores resultados. Fatores socioeconômicos, problemas para a fixação de profissionais e baixo processo formativo são fatores que contribuem para esse panorama desfavorável ${ }^{15,16}$.

De modo geral, estudos nacionais ${ }^{7,9,17,18}$ demonstram significativa melhoria da estruturação da APS ao longo do tempo e que esse processo possibilita a inserção de ações em saúde mental no nível primário, o que, por sua vez, promove melhora do cuidado

Fatores como o enfrentamento aos desafios da reforma psiquiátrica, a criação e ampliação de serviços, a instituição da RAPS, ampliação e definição da APS como prioritária para o cuidado e ter ampla diversidade de serviços substitutivos são avanços significativos para a saúde mental e a atenção primária ${ }^{7,19,20}$, porém é preciso ainda evoluir na inserção de ações em SM na APS.

Há controvérsias nos diversos estudos sobre a estruturação dos atributos na APS. Pesquisas usando o PCAtool ressaltam que o primeiro contato é um dos atributos mais frágeis da $\mathrm{APS}^{21,22}$. No entanto, outro estudo, utilizando dados do PMAQ, com dados das ESF, unidades e usuários, apontou a longitudinalidade e a coordenação como os atributos pior avalidados ${ }^{19}$. Este estudo, porém, evidencia que os atributos atuam de forma diferente em cenários de alta e baixa estruturação da APS. O maior número de ESF no melhor cenário possui o atributo longitudinalidade estruturado; já para o pior cenário, o atributo coordenação é que necessita ser estruturado.

Os resultados das análises de associação entre a qualidade da assistência e a estruturação da APS demonstram uma associação positiva que amplia em 14 vezes a chance de a APS prestar melhores serviços em saúde mental quando as EqAB estão mais bem estruturadas. Nesse sentido, as equipes devem se preparar para absorver o cuidado em SM, com capacitação dos profissionais, mudanças nos processos de trabalho das equipes e adequação de estrutura física para o enfrentamento desse grande desafio ${ }^{7}$.

É preciso levar em consideração que a inserção de ações em saúde mental também sofra resistência por parte dos profissionais, que precisam incorporar os princípios do modelo substitutivo, em que as EqAB priorizam os transtornos mentais da mesma forma que faz com outras doenças ${ }^{4,23,24}$. Ao focalizar a saúde mental no processo de estruturação da APS, esta pesquisa constata o quanto a área de SM ainda precisa avançar em seu processo de organização, acompanhando o fortalecimento da atenção primária no país.

Embora a adesão ao $2^{\circ}$ ciclo do PMAQ-AB tenha sido bastante elevada, a análise apenas das equipes que aderiram voluntariamente pode superestimar alguns resultados, o que se apresenta com uma limitação para este estudo. O vínculo do PMAQ-AB com recursos financeiros de suporte ao programa também pode comprometer os resultados encontrados. O instrumento de coleta de dados do PMAQ-AB também apresenta limites na formulação de alguns quesitos na área de saúde mental, como na caracterização de subgrupos investigados (usuários de drogas e de medicamentos psicotrópicos) e de algumas ações ofertadas. Também outros aspectos relativos ao atendimento em grupo, ações de prevenção e de promoção da saúde mental não se encontram adequadamente estruturados no PMAQ-AB. Portanto, a oferta de cuidado em saúde mental, ainda que entendida como parte do escopo de ações da 
ABS, mostra-se pouco desenvolvida. Apesar desses limites, os dados advindos do PMAQ-AB permitem uma visão de como a área se encontra estruturada na APS no Brasil.

\section{CONCLUSÕES}

A qualidade do cuidado em SM na APS no Brasil ainda tem um longo percurso à frente, mais de um terço das EqAB possui qualidade ruim de estruturação das ações. A atenção à saúde mental não acompanha de forma linear a estruturação da APS, exigindo esforços adicionais para sua implementação. Observou-se que os atributos essenciais que mais contribuem para melhor oferta de cuidados com boa qualidade são integralidade e coordenação.

Historicamente, o movimento de saúde mental tem estruturado a rede de atenção centrada em equipamentos substitutivos. Atualmente, com as políticas de saúde mental enfrentando retrocessos que podem ser catastróficos, inserir ações de SM na APS no Brasil torna-se ainda mais importante. Assim, a consideração de tais aspectos pode contribuir de forma marcante para a efetivação do cuidado em saúde mental com qualidade e excelência.

\section{REFERÊNCIAS}

1. Kohn R, Ali AA, Puac-Polanco V, Figueroa C, López-Soto V, Morgan K, et al. Mental health in the Americas: an overview of the treatment gap. Rev Panam Salud Publica. 2018;42:e165. https://doi.org/10.26633/RPSP.2018.165

2. Bonadiman CSC, Passos VMA, Mooney M, Naghavi M, Melo APS. A carga dos transtornos mentais e decorrentes do uso de substâncias psicoativas no Brasil: Estudo de Carga Global de Doença, 1990 e 2015. Rev Bras Epidemiol. 2017;20 Supl 1:191-204. https://doi.org/10.1590/1980-5497201700050016

3. Mulvaney-Day N, Marshall T, Downey Piscopo K, Korsen N, Lynch S, Karnell LH, et al. Screening for behavioral health conditions in primary care settings: a systematic review of the literature. J Gen Intern Med. 2018;33:335-46. https://doi.org/10.1007/s11606-017-4181-0

4. Souza LGS, Menandro MCS, Couto LLM, Schimith PB, Lima RP. Saúde mental na Estratégia Saúde da Família: revisão da literatura brasileira. Saude Soc. 2012;21(4):1022-34. https://doi.org/10.1590/S0104-12902012000400019

5. Moliner J, Lopes SMB. Saúde mental na atenção básica: possibilidades para uma prática voltada para a ampliação e integralidade da saúde mental. Saude Soc. 2013;22(4):1072-83. https://doi.org/10.1590/S0104-12902013000400010

6. Rocha BS, Werlang MC. Psicofármacos na Estratégia Saúde da Família: perfil de utilização, acesso e estratégias para a promoção do uso racional. Cienc Saude Coletiva. 2013;18(11):3291-300. https://doi.org/10.1590/S1413-81232013001100019

7. Gerbaldo TB, Arruda AT, Horta BL, Garnelo L. Avaliação da organização do cuidado em Saúde Mental na Atenção Básica à Saúde do Brasil. Trab Educ Saude. 2018;16(3):1079-94. https://doi.org/10.1590/1981-7746-sol00150

8. Silva Filho JA, Bezerra AM. Acolhimento em Saúde Mental na Atenção Primária à Saúde: revisão Integrativa. Id on Line Rev Multidiscipl Psicol. 2018 [citado 31 jan 2020];12(40). Disponível em: https://idonline.emnuvens.com.br/id/article/view/1138/0

9. Frateschi MS, Cardoso CL. Práticas em saúde mental na atenção primária à saúde. Psico (Porto Alegre). 2016;47(2):159-68. https://doi.org/10.15448/1980-8623.2016.2.22024

10. Medrado ACC, Cruz MG, Baião JJ, Souza MA, Araújo PS. Os laços e nós de uma rede de atenção psicossocial. Cad Bras Saude Mental. 2018;10(27):53-70.

11. Bezerra IC, Jorge MSB, Gondim APS, Lima LL, Vasconcelos MGF. “Fui lá no posto e o doutor me mandou foi pra cá": processo de medicamentalização e (des)caminhos para o cuidado em saúde mental na Atenção Primária. Interface. 2014;18(48):61-74. https://doi.org/10.1590/1807-57622013.0650

12. Correia VR, Barros S, Colvero LA. Saúde mental na atenção básica: prática da equipe de saúde da família. Rev Esc Enferm USP. 2011;45(6):1501-6. https://doi.org/10.1590/S0080-62342011000600032 
13. Machado DKS, Camata MW. Apoio matricial como ferramenta de articulação entre a saúde mental e a atenção primária à saúde. Cad Saude Coletiva. 2013;22(2):224-32.

14. Quinderé PHD, Jorge MSB, Nogueira MSL, Costa LFA, Vasconcelos MGF. Acessibilidade e resolubilidade da assistência em saúde mental: a experiência do apoio matricial. Cienc Saude Coletiva. 2013;18(7):2157-66. https://doi.org/10.1590/S1413-81232013000700031

15. Lima JG, Giovanella L, Fausto MCR, Bousquat A. Qualidade da atenção básica por tipos de regiões de saúde: nota técnica. Novos Caminhos. 2016 [citado 17 jan 2020];(12):1-76. Disponível em: https://www.resbr.net.br/wp-content/uploads/2016/07/NovosCaminho12.pdf

16. Fausto MCR, Giovanella L, Mendonça MHM, Seidl H, Gagno J. A posição da Estratégia Saúde da Família na rede de atenção à saúde na perspectiva das equipes e usuários participantes do PMAQ-AB, 2012. Saude Debate. 2014;38 Nº Espec:13-33. https://doi.org/10.5935/0103-1104.2014S003

17. Chaves LA, Jorge AO, Cherchiglia ML, Reis IA, Santos MAC, Santos AF, et al. Integração da atenção básica à rede assistencial: análise de componentes da avaliação externa do PMAQ-AB. Cad Saude Publica. 2018;34(2):e00201515. https://doi.org/10.1590/0102-311X00201515

18. Cavalli LO, Rizzotto MLF, Guimarães ATB. O médico no processo de avaliação externa do Programa Nacional de Melhoria do Acesso e da Qualidade da Atenção Básica, ciclos I e II. Saude Debate. 2016;40(111):87-100. https://doi.org/10.1590/0103-1104201611107

19. Santos AB, Silva GG, Pereira MER, Brito RS. Saúde mental, humanização e direitos humanos. Cad Bras Saude Mental. 2018;10(25):1-19. Segundo volume da Edição Especial III Fórum de Direitos Humanos e Saúde Mental.

20. Amarante $P$, Nunes MO. A reforma psiquiátrica no SUS e a luta por uma sociedade sem manicômios. Cienc Saude Coletiva. 2018;23(6):2067-74. https://doi.org/10.1590/1413-81232018236.07082018

21. Silva GS, Alves CRL. Avaliação do grau de implantação dos atributos da atenção primária à saúde como indicador da qualidade da assistência prestada às crianças. Cad Saude Publica. 2019;35(2):e00095418 https://doi.org/10.1590/0102-311X00095418

22. Prates ML, Machado JC, Silva LS, Avelar PS, Prates LL, Mendonça ET, et al. Desempenho da Atenção Primária à Saúde segundo o instrumento PCATool: uma revisão sistemática. Cienc Saude Coletiva. 2017;22(6):1881-93. https://doi.org/10.1590/1413-81232017226.14282016

23. Campos RTO, Ferrer AL, Gama CAP, Campos GWS, Trapé TL, Dantas DV. Avaliação da qualidade do acesso na atenção primária de uma grande cidade brasileira na perspectiva dos usuários. Saude Debate. 2014;38 № Espec:252-64. https://doi.org/10.5935/0103-1104.2014S019

24. Mendonça MHM, Matta GC, Gondim R, Giovanella L, organizadores. Atenção Primária à Saúde no Brasil: conceitos, práticas e pesquisa. Rio de Janeiro: Fiocruz; 2018. Introdução; p. 29-47.

Contribuição dos Autores: Concepção e planejamento do estudo: AMJL, AFS, EIGA. Coleta, análise e interpretação dos dados: AMJL, AFS, EIGA. Elaboração ou revisão do manuscrito: AMJL, AFS, EIGA, ATMM. Aprovação da versão final: AMJL, AFS, EIGA, ATMM. Responsabilidade pública pelo conteúdo do artigo: |AMJL, AFS, EIGA, ATMM.

Conflito de Interesses: Os autores declaram não haver conflito de interesses. 